\title{
Decay of Neutron-Rich Mn Nuclides and Deformation of Heavy Fe Isotopes
}

\author{
M. Hannawald, ${ }^{1}$ T. Kautzsch, ${ }^{1}$ A. Wöhr, ${ }^{2}$ W. B. Walters, ${ }^{3}$ K.-L. Kratz, ${ }^{1}$ V. N. Fedoseyev, ${ }^{4}$ V. I. Mishin, ${ }^{4}$ W. Böhmer,${ }^{1}$

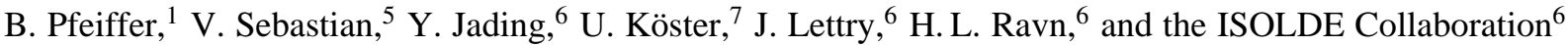 \\ ${ }^{1}$ Institut für Kernchemie, Universität Mainz, D-55099 Mainz, Germany \\ ${ }^{2}$ Instituut voor Kern- en Strahlingsfysica, University of Leuven, B-3001 Leuven, Belgium \\ ${ }^{3}$ Department of Chemistry, University of Maryland, College Park, Maryland 20742 \\ ${ }^{4}$ Institute of Spectroscopy, Russian Academy of Sciences, RUS-142092 Troitzk, Russia \\ ${ }^{5}$ Institut für Physik, Universität Mainz, D-55099 Mainz, Germany \\ ${ }^{6}$ CERN, CH-1211 Geneva 23, Switzerland \\ ${ }^{7}$ Physik-Department, TU München, D-85748 Garching, Germany
}

(Received 13 March 1998)

\begin{abstract}
The use of chemically selective laser ionization combined with $\beta$-delayed neutron counting at CERN/ISOLDE has permitted identification and half-life measurements for $623-\mathrm{ms}{ }^{61} \mathrm{Mn}$ up through $14-\mathrm{ms}{ }^{69} \mathrm{Mn}$. The measured half-lives are found to be significantly longer near $N=40$ than the values calculated with a quasiparticle random-phase-approximation shell model. Gamma-ray singles and coincidence spectroscopy has been performed for ${ }^{64,66} \mathrm{Mn}$ decays to levels of ${ }^{64,66} \mathrm{Fe}$, revealing a significant drop in the energy of the first $2^{+}$state in these nuclides that suggests an unanticipated increase in collectivity near $N=40 . \quad$ [S0031-9007(99)08463-X]
\end{abstract}

PACS numbers: 21.10.Tg, 23.20.Lv, 23.40.Hc, 27.50.+e

Until recently, the principal data available for neutronrich nuclides between ${ }^{48} \mathrm{Ca}$ and ${ }^{78} \mathrm{Ni}$ were the $\gamma$ spectroscopic data obtained in the 1980s at Gesellschaft für Schwerionenforschung (GSI) $[1,2]$ and the nuclear masses reported by Seifert et al. [3]. During the past three years, however, a number of new experimental studies on level structures and decay properties on Fe-group nuclei have been performed [4-9]. On the theoretical side, nuclear masses, ground-state (g.s.) deformations, and $\beta$-decay properties were calculated by Möller et al. [10] on the basis of the finite-range droplet mass model (FRDM) and the quasiparticle random-phaseapproximation (QRPA) shell model. Aboussir et al. [11] also calculated masses and g.s. deformations, on the basis of the extended Thomas-Fermi plus Strutinsky-integral (ETFSI) approach, which are not always in agreement with those of the FRDM for the Fe-group nuclides considered here. Earlier, Richter et al. [12] performed fully microscopic shell-model calculations in this mass region and significantly underpredicted the measured [3] binding energies for the $\mathrm{Cr}$ to $\mathrm{Fe}$ isotopes with $N>36$. In addition to the clear nuclear-structure interest, the neutron-rich Fe-group nuclei may also play an important role as possible seed nuclei in the astrophysical $r$ process [13]. In the present paper, we report new measurements for the half-lives of heavy $\mathrm{Mn}$ nuclides up to ${ }^{69} \mathrm{Mn}$ and for the level structure of ${ }^{64,66} \mathrm{Fe}$ populated in the decays of ${ }^{64,66} \mathrm{Mn}$.

Manganese isotopes were produced at CERN by $1-\mathrm{GeV}$ proton-induced spallation of uranium in a thick $\mathrm{UC}_{2}$ target at the ISOLDE facility. The ionization of the Mn atoms was accomplished using a chemically selective, three-step laser resonance excitation scheme as described in detail earlier [14].
Beams of Mn nuclides with masses differing by $\Delta A \geq$ 4 were transported separately to two different beam lines equipped with moving tape systems where $\beta$-delayed neutron (d.n.) multiscaling and $\gamma$-ray singles and coincidence measurements could be performed independently. In both cases, counting took place directly at the point of deposit, and the tape systems were used to remove the daughter nuclides as well as unavoidable surfaceionized isobaric $\mathrm{Ga}$ activities. Because the Mn half-lives being sought are in the millisecond range, data acquisition in both systems was initiated by the proton pulses from the CERN proton-synchrotron booster (PSB), separated by a multiple of $1.2 \mathrm{~s}$, and continued for $1.0 \mathrm{~s}$ for each cycle.

Beta-delayed neutron data of high statistical quality were collected by multiscaling measurements using the Mainz $4 \pi^{3} \mathrm{He}$ neutron counter. The time dependence of the counting rates for ${ }^{65-69} \mathrm{Mn}$ is shown in Fig. 1. The decay curves were fitted with a constant small d.n.background component up through $A=65$. Because there exist no measured d.n.-emission probabilities ( $P_{n}$ values) for the $A>65$ daughter and granddaughter isobars, the fits of the heavier isotopes were performed using theoretical $P_{n}$ values [10] along with the known half-lives $[5,7,8,15]$. For $A=66-68$, the contributions from d.n. emission of the $\mathrm{Fe}$ and $\mathrm{Co}$ isobars are quite small and actually do not affect the Mn half-life fits. For $A=69$, however, a multicomponent fit was necessary to account for the significant $\mathrm{Fe}$ and Co d.n. branches. The resulting data are summarized in Table I, and are compared to literature (lit.) values and QRPA predictions using experimental masses as far as they are available [15] and g.s. deformations of the $\beta$ decay daughter isotopes from the FRDM [10] and ETFSI [11] models. 

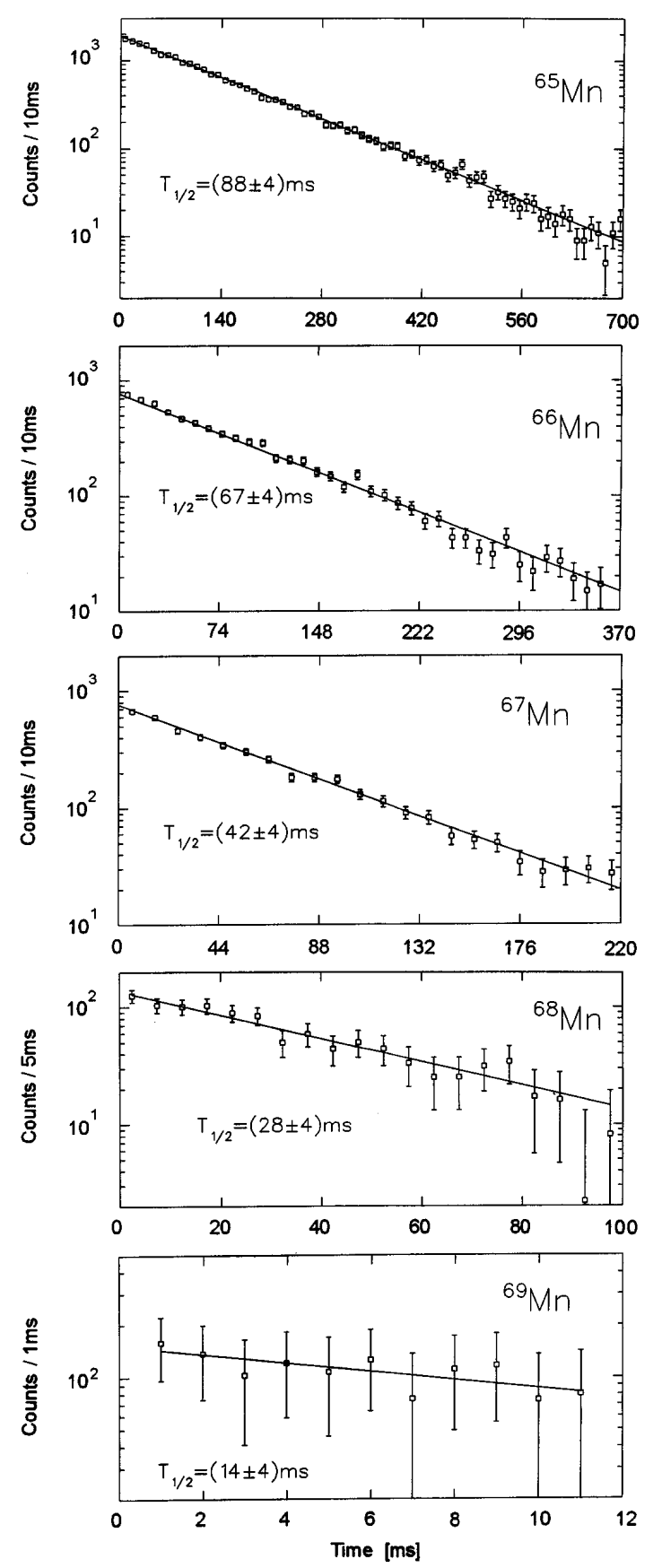

FIG. 1. Beta-delayed neutron decay curves for the neutronrich isotopes ${ }^{65-69} \mathrm{Mn}$.

For ${ }^{61-63} \mathrm{Mn}$, the half-lives that we have observed are somewhat shorter than the literature values and have considerably smaller uncertainties. In a recent report of a parallel experiment at Ganil, Sorlin et al. [5] found halflives similar to ours for ${ }^{64-66} \mathrm{Mn}$. However, neither our nor their data are in agreement with those reported by Ameil et al. at ENAM'95 [7] and cited in NUBASE [15]. In a subsequent publication the same authors report somewhat shorter half-lives for these nuclides [16], however, still exceeding our and Sorlin's values. Similarly, Fran-
TABLE 1. Experimental and theoretical half-lives for the neutron-rich Mn nuclides. In the QRPA calculations, deformation values $\beta_{2}(\mathrm{Fe})$ were taken from FRDM [10] and ETFSI [11].

\begin{tabular}{cccrcc}
\hline \hline Mass & \multicolumn{3}{c}{ Half-life (ms) } & \multicolumn{2}{c}{} \\
& This work & Lit. & Ref. & \multicolumn{2}{c}{ QRPA } \\
& & & & {$[10]$} & {$[11]$} \\
\hline 61 & $623(10)$ & $719(10)$ & {$[2]$} & 234 & 231 \\
62 & $671(5)$ & $880(150)$ & {$[15]$} & 267 & 274 \\
63 & $275(4)$ & $282(18)$ & {$[15]$} & 76 & 76 \\
$\ldots$ & $\ldots$ & $321(22)$ & {$[5]$} & $\ldots$ & $\ldots$ \\
64 & $89(4)$ & $240(30)$ & {$[7]$} & 67 & 105 \\
$\ldots$ & $\ldots$ & $140(30)$ & {$[16]$} & $\ldots$ & $\ldots$ \\
$\ldots$ & $\ldots$ & $91(7)$ & {$[5]$} & $\ldots$ & $\ldots$ \\
65 & $88(4)$ & $160(30)$ & {$[7]$} & 39 & 46 \\
$\ldots$ & $\ldots$ & $110(20)$ & {$[16]$} & $\ldots$ & $\ldots$ \\
$\ldots$ & $\ldots$ & $88(7)$ & {$[5]$} & $\ldots$ & $\ldots$ \\
66 & $66(4)$ & $220(40)$ & {$[7]$} & 21 & 23 \\
$\ldots$ & $\ldots$ & $90(20)$ & {$[16]$} & $\ldots$ & $\ldots$ \\
$\ldots$ & $\ldots$ & $62(13)$ & {$[5]$} & $\ldots$ & $\ldots$ \\
67 & $42(4)$ & $\ldots$ & $\ldots$ & 25 & 27 \\
68 & $28(4)$ & $\ldots$ & $\ldots$ & 18 & 25 \\
69 & $14(4)$ & $\ldots$ & $\ldots$ & 16 & 14 \\
\hline \hline
\end{tabular}

choo et al. found systematic differences between their $\mathrm{Ni}$ half-lives [8] and those reported in [7,16]. These deviations gain particular importance when considering the conclusion of Ameil et al. that recent theoretical half-lives are not an improvement over calculations made almost a decade ago [16].

A comparison of the experimental Mn half-lives with the predictions derived from QRPA calculations of Gamow-Teller (GT) strength functions [17] (see Table I) indicates that the theoretical half-lives for the g.s. decays of ${ }^{61-66} \mathrm{Mn}$ are, on average, shorter than the measured ones by a factor of 2.6, whereas for the heaviest isotopes ${ }^{67-69} \mathrm{Mn}$ the agreement becomes gradually better. When looking in more detail into the theoretical $\beta$-strength distributions, it becomes evident that, in the decay of all neutron-rich $\mathrm{Mn}$ isotopes considered here, the (low-lying) $\nu f_{5 / 2} \rightarrow \pi f_{7 / 2}$ transition strongly dominates the GT decay with $\mathrm{I}_{\beta} \simeq 85-95 \%$ and $\log (f t) \simeq 4.0$, practically independent of the assumed g.s. shape of the Fe daughters $[10,11]$. As will be discussed later, this GT pattern is, indeed, observed in the $\gamma$ data of ${ }^{64} \mathrm{Mn}$ and ${ }^{66} \mathrm{Mn}$ decay. With this rather "simple" decay pattern, already the differences between the experimental and theoretical half-lives seem to reflect the actual strength of this specific spin-flip transition.

The $\gamma$-ray data were written in an event-by-event mode for $\beta$-gated $\gamma$ singles as well as $\gamma \gamma(\mathrm{t})$ coincidences with time recorded relative to each proton pulse. In this way, consecutive spectra of variable time intervals could be reconstructed from the data. As considerable data exist for the structure of even-even Fe nuclides up to $A=62$, 
in the present study we have focused on the $\gamma$ spectra for decay of 89-ms ${ }^{64} \mathrm{Mn}$ and $66-\mathrm{ms}{ }^{66} \mathrm{Mn}$.

At $A=64, \gamma$-ray peaks up to $4.2 \mathrm{MeV}$ could be assigned to the decay of ${ }^{64} \mathrm{Mn}$. More than 20 lines have been incorporated into a decay scheme of at least eight excited levels [18]. Partial $\gamma$-ray spectra are shown in the upper part of Fig. 2, one for the time period from 40 to $140 \mathrm{~ms}$ after the PSB proton pulses, and a second for the time slice from 800 to $900 \mathrm{~ms}$ after bombardment. The most intense line in the early ${ }^{64} \mathrm{Mn}$ spectrum that decays with a short half-life is at $746 \mathrm{keV}$. With the intensity of this $\gamma$ line being more than 5 times stronger than the next most intense peak, it is taken to be the $2^{+}$to $0^{+}$transition in the even-even daughter ${ }^{64} \mathrm{Fe}$. As five of the eight levels appear to depopulate to both the g.s. and the first $2^{+}$level, a low spin for the g.s. of ${ }^{64} \mathrm{Mn}$ is indicated. No candidate has so far been identified unambiguously for the $4^{+}$to $2^{+}$ transition. On the basis of the observed $\gamma \gamma$ coincidences, strong GT feeding to levels near $3.5 \mathrm{MeV}$ in ${ }^{64} \mathrm{Fe}$ is indicated [18], as predicted by our QRPA calculations.

More than $20 \gamma$-ray lines with energies up to at least 4.2 MeV have also been observed in the decay of 66$\mathrm{ms}{ }^{66} \mathrm{Mn}$ and incorporated into a partial level scheme containing 11 levels. We show in the lower part of Fig. 2 a portion of the first and of a late spectrum (see above) between 525 and $900 \mathrm{keV}$. In the early spectrum, the strongest line by far is at $573 \mathrm{keV}$ which is - as in the above case of ${ }^{64} \mathrm{Fe}$ - taken as the g.s. transition from the first $2^{+}$state in even-even ${ }^{66} \mathrm{Fe}$. The relatively weak $\gamma$ line at $840 \mathrm{keV}$ (also shown in Fig. 2) is found in

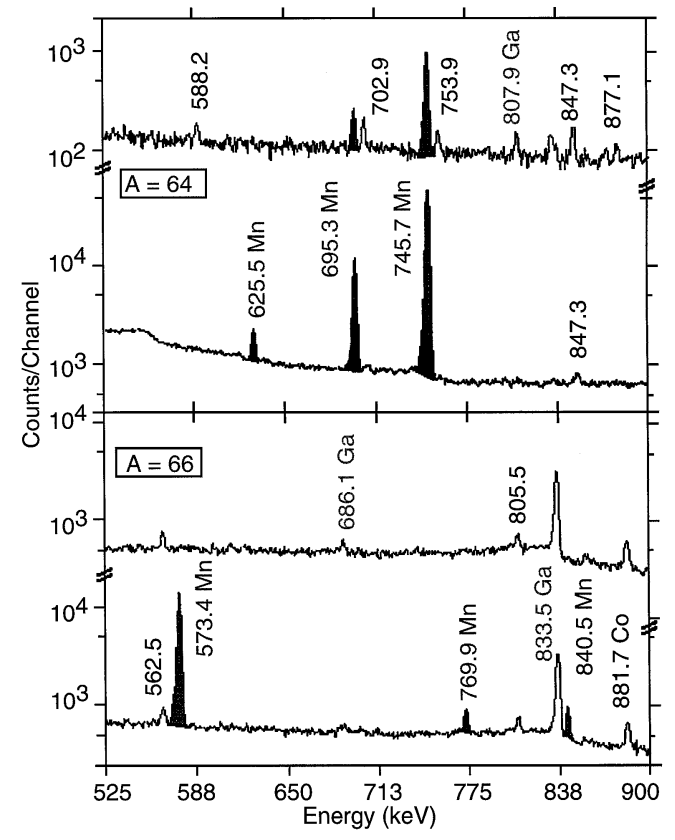

FIG. 2. Partial $\gamma$-ray spectra taken at $A=64$ (upper part) and $A=66$ (lower part), one for the time period from 40 to $140 \mathrm{~ms}$ after the PSB proton pulses, and a second for the time slice from 800 to $900 \mathrm{~ms}$ after bombardment. coincidence with the 573-keV level and decays with the same half-life. Hence, it is assigned as depopulating the second excited state at $1414 \mathrm{keV}$ and given a tentative spin and parity of $4^{+}$. A number of high-energy g.s. transitions $(E>2.7 \mathrm{MeV})$ again indicate strong GT feeding to that energy region [18], as predicted by the QRPA.

At both $A=64$ and 66 , it was also possible to identify the growth and decay of known daughter and granddaughter $\mathrm{Fe}$ and $\mathrm{Co}$ lines; whereas the lines from long-lived, surface-ionized ${ }^{64} \mathrm{Ga}$ and ${ }^{66} \mathrm{Ga}$ showed no change in intensity in our $1.0 \mathrm{~s}$ measuring period.

The $2_{1}^{+}$energies and the $E_{4} / E_{2}$ ratios for even-even ${ }_{24} \mathrm{Cr}$ to ${ }_{32} \mathrm{Ge}$ isotopes are shown in Fig. 3. As is well established, the $2^{+}$energy in the $Z=28 \mathrm{Ni}$ nuclides rises sharply at $N=40$ and exhibits clear evidence for a semidouble shell closure, similar to that for $Z=40, N=50$ in ${ }^{90} \mathrm{Zr}$. Recent studies of the structure of ${ }^{69} \mathrm{Ni}$ and ${ }^{69} \mathrm{Cu}$ are consistent with the closed-shell character of ${ }^{68} \mathrm{Ni}[8,9]$.

Raman et al. [19] have presented an extensive discussion of the relationship between $2^{+}$energies, $B(E 2)$ values, and collectivity. In particular, they have shown the inverse correlation between $2^{+}$energies and deformation. Similar correlations of $B(E 2)$ values with energy have recently been presented by Azaiez and Sorlin [20]. Hence, the direct interpretation of the drop in $2^{+}$energy from $877 \mathrm{kev}$ in ${ }^{62} \mathrm{Fe}$ to $573 \mathrm{kev}$ in ${ }^{66} \mathrm{Fe}$ would be an increase in deformation. Because of the large quantity of data available, including the $B(E 2)$ systematics [19], there is general agreement on deformation values of $\beta_{2} \simeq 0.18$ for the lighter Fe nuclides. For the heavier isotopes where only few data are available, both the FRDM [10] and new calculations using the relativistic mean field approach [21] indicate deformation of $\sim 0.21$ for ${ }^{62} \mathrm{Fe}$ and then dropping toward values below 0.1 for ${ }^{66} \mathrm{Fe}$. In contrast, the ETFSI calculations [11] show a value of $\beta_{2}=0.18$ for ${ }^{62} \mathrm{Fe}$ that rises to 0.27 for ${ }^{66} \mathrm{Fe}$.

The data in Fig. 3 reveal that both the $2_{1}^{+}$energy and $4^{+} / 2^{+}$ratio for ${ }^{66} \mathrm{Fe}_{40}$ are comparable to those of stable ${ }^{75} \mathrm{Ge}_{44}$. Extensive studies of the structure of the stable Ge nuclides [22,23] have indicated a range of deformation values between $\beta_{2}=0.22$ and 0.28 for ${ }^{76} \mathrm{Ge}$. Azaiez and Sorlin recently reported a $\beta_{2}$ value of 0.23 for ${ }^{72} \mathrm{Zn}_{42}$ that has a $2^{+}$energy of $653 \mathrm{keV}$ [20]. Combining all of the above approaches, we deduce a $\beta_{2} \simeq 0.26$ for ${ }^{66} \mathrm{Fe}_{40}$. Thus, we conclude that the trends we observe for the $\mathrm{Fe}$ isotopes are reproduced only by the ETFSI calculations [11].

We attribute this increase of deformation to the strong proton-neutron ( $\mathrm{pn}$ ) interaction between the two $f_{7 / 2}$ proton holes and the $g_{9 / 2}$ neutrons, which results in a dramatic lowering of the energy of the $\nu g_{9 / 2}$ orbital. The effect of interactions between protons and neutrons in high-j orbitals has also been discussed in [8] for oddmass $\mathrm{Cu}$ nuclides near $N=40$. In that paper, a sharp lowering of the energy of the $\pi f_{5 / 2}$ orbital was observed with increasing occupancy of the $\nu g_{9 / 2}$ state. 

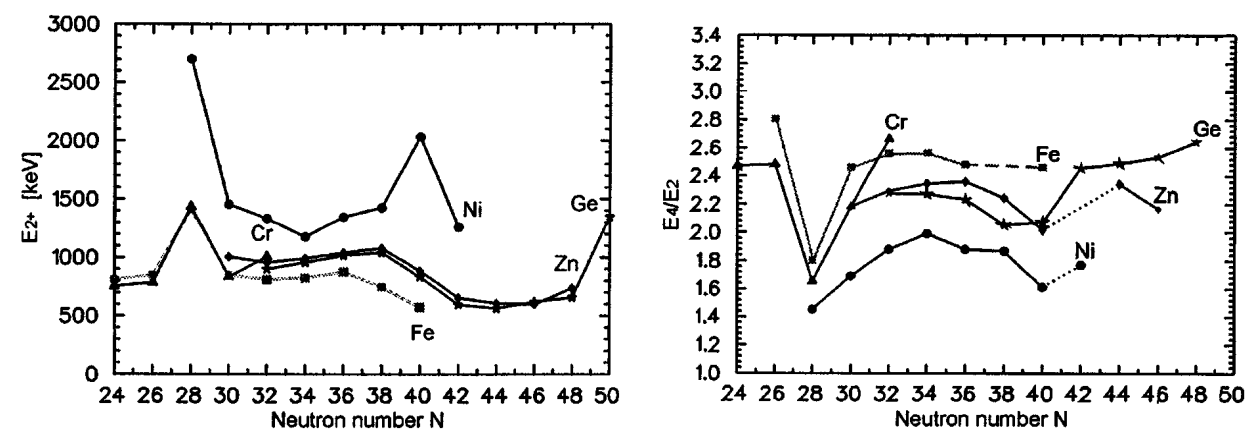

FIG. 3. Energies of the first $2^{+}$levels and $E_{4+} / E_{2+}$ ratios of even-even ${ }_{24} \mathrm{Cr}$ to ${ }_{32} \mathrm{Ge}$ nuclides.

For the neutron-rich $\mathrm{Fe}$ and $\mathrm{Mn}$ nuclides, one effect of this strong pn interaction is to lower the energy of the $\nu g_{9 / 2}$ orbital. For example, the recent report of a $9 / 2^{+} M 2$ isomer in ${ }^{61} \mathrm{Fe}_{35}$ at $861 \mathrm{keV}$ is well below the $1292-\mathrm{keV}$ position of the $\nu g_{9 / 2}$ state in isotonic ${ }^{63} \mathrm{Ni}_{35}$ [9]. Because of the lowered position of the base $\nu g_{9 / 2}$ orbital, it can be seen by reference to Fig. 12 in [10] or Fig. 7 in [5] that, as the neutron number increases beyond 36, the down-sloping $\nu[440] 1 / 2^{+}$and $\nu[431] 3 / 2^{+}$orbitals are more likely to be occupied than the spherical orbitals, thus generating increased deformation at $N=40$. This collectivity increase beyond $N=36$ can be seen to correlate with the difference between the masses calculated with a spherical model and the measured values shown by Richter et al. [12] in their Fig. 1. From the trends in that figure, it can be expected that ${ }^{64} \mathrm{Cr}_{40}$ may be even more deformed than ${ }^{66} \mathrm{Fe}_{40}$.

Moreover, such a change in neutron occupancy near $N=40$ may help to account for the retarded $\mathrm{Mn} \beta$ decay rates described earlier. The decay of the odd-mass Mn nuclides would be considered as "even-jumping" transitions as described by Kisslinger and Sorensen [24]. For such transitions, the $\beta$-decay rate $\lambda$ is proportional to $\left(V_{n} \cdot V_{p}\right)^{2} \cdot M^{2}$, where $M^{2}$ is the pure decay matrix element and $V$ represents the proton/neutron occupancy values. As noted earlier, the allowed GT $\beta$ decay is dominated by the $\nu f_{5 / 2} \rightarrow \pi f_{7 / 2}$ transition. Because of the increase in deformation, the negative parity orbitals $\nu[301] 3 / 2^{-}$and $\nu[303] 5 / 2^{-}$with $\nu f_{5 / 2}$ parentage are up-sloping and are thus displaced from the region near the Fermi surface. Instead, the 37th through 40th neutrons will preferentially fill the down-sloping low-j, positiveparity orbitals with a $\nu g_{9 / 2}$ origin that can only undergo forbidden $\beta$ decay. The lowered occupancy of the above critical negative-parity states may well be directly responsible for the observed retardation of the $\beta$-decay rates for $\mathrm{Mn}$ nuclides with $N \simeq 40$. Moreover, the ultimate increase in occupancy for these orbitals that must occur for $N>40$ also accounts for the gradual convergence of the calculated half-lives and the newly measured values for ${ }^{67-69} \mathrm{Mn}_{42-44}$.
This work was supported by the German BMBF (06MZ864) and DFG (436RUS/17/40/97 and Kr806/ $3)$, the Russian Foundation for Basic Research (96-0218331), the Belgian FWO, and the U.S. DOE.

[1] U. Bosch et al., Phys. Lett. 164B, 22 (1985); Nucl. Phys. A477, 89 (1988).

[2] E. Runte et al., Nucl. Phys. A399, 163 (1983).

[3] H. L. Seifert et al., Z. Phys. A 349, 25 (1994).

[4] T. Pawlat et al., LNL 1995 Prog. Rep., LNL-INFN 105/ 96, 7 (1996); R. Broda et al., Il Nuovo Cimento (to be published).

[5] O. Sorlin et al., in Proceedings of the ENAM'95 Conference (Editions Frontieres, Gif-sur-Yvette, 1995) p. 603; Nucl. Phys. A632, 205 (1998); Il Nuovo Cimento (to be published).

[6] T. Dörfler et al., Phys. Rev. C 54, 2894 (1996).

[7] F. Ameil et al., Proceedings of the ENAM'95 Conference (Editions Frontieres, Gif-sur-Yvette, 1995), p. 537.

[8] S. Franchoo et al., Phys. Rev. Lett. 81, 3100 (1998).

[9] R. Grzywacz et al., Phys. Rev. Lett. 81, 766 (1998).

[10] P. Möller et al., At. Data Nucl. Data Tables 59, 183 (1995); 66, 131 (1997).

[11] Y. Aboussir et al., At. Data Nucl. Data Tables 61, 127 (1995).

[12] W. A. Richter et al., Nucl. Phys. A586, 445 (1995).

[13] K. Takahashi et al., Astron. Astrophys. 286, 857 (1994).

[14] V.I. Mishin et al., Nucl. Instrum. Methods Phys. Res., Sect. B 73, 550 (1993); 126, 88 (1997).

[15] G. Audi et al., Nucl. Phys. A624, 1 (1997).

[16] F. Ameil et al., Eur. Phys. J. A 1, 275 (1998).

[17] P. Möller and J. Randrup, Nucl. Phys. A514, 1 (1990).

[18] M. Hannawald et al., (to be published).

[19] S. Raman, C. W. Nestor, S. Kahane, and K. H. Bhatt Phys. Rev. C 43, 556 (1991)

[20] F. Azaiez and O. Sorlin, Nucl. Phys. News 8, 34 (1998).

[21] D. Hirata et al., Nucl. Phys. A616, 438c (1997).

[22] R. Lecomte et al., Phys. Rev. C 22, 1530 (1980); 22, 2420 (1980).

[23] M. Huhta et al., Phys. Rev. C 58, 3187 (1998).

[24] L. S. Kisslinger and R. A. Sorensen, Rev. Mod. Phys. 35, 853 (1963). 